$$
\left(\begin{array}{cc}
\|Z\| & 0 \\
0 & I
\end{array}\right)
$$

It is possible that these together with the constant unitary matrices generate the whole class of such functions, but we have not been able to prove it.

\title{
REFERENCES
}

1. I. Priwalow, Randeigenschaften Analytisches Functionen, VEB Deutscher Verlag Wissenschaften, Berlin, 1956.

2. V. P. Potapov, The multiplicative structure of J-contractive matrix functions, Amer. Math. Soc. Transl. (2) 15 (1960), 131-243.

Institute for Defense Analyses, Princeton, New Jersey

\section{ON THE RADIUS OF UNIVALENCE OF CERTAIN ANALYTIC FUNCTIONS}

\section{A. E. LIVINGSTON}

Let $\mathfrak{C}$ denote the class of functions $f$ regular and univalent in $E=\{z|| z \mid<1\}$, which satisfy $f(0)=0$ and $f^{\prime}(0)=1$ and which are close-to-convex in $E$. Let $\mathcal{K}$ and $\mathcal{S}^{*}$ denote the subfamilies of $\mathcal{C}$, made up of functions which are convex and starlike in $E$, respectively. Recently, Libera [2] has shown that if $f$ is a member of $\Re$, $\mathcal{S}^{*}$ or $\mathfrak{e}$, then the function $F(z)=(2 / z) \int_{0}^{z} f(t) d t$ is also a member of $\Re, \varsigma^{*}$ or $\mathcal{C}$. It is the purpose of this paper to investigate the converse question. That is, if $F$ is in $\delta^{*}$, what is the radius of starlikeness of the function $f(z)=[1 / 2][z F(z)]^{\prime}$ ? Similar questions are answered under the assumption that $F$ is in $\nVdash$ or in $\mathcal{C}$. Robinson [5] has shown that if $F$ is only assumed to be univalent in $E$, then $f$ is starlike for $|z|<.38$. He pointed out that it is probable that $f$ is univalent for $|z|<(1 / 2)$. We obtain this result under the added assumption that $F$ is a member of $\Re, s^{*}$ or $\mathcal{C}$.

The method of proof used in Theorem 1 has recently been employed by MacGregor [4].

Presented to the Society, December 6, 1965; received by the editors April 28, 1965. 
THEOREM 1. If $F$ is in $\delta^{*}$, then $f(z)=[1 / 2][z F(z)]^{\prime}$ is starlike for $|z|<1 / 2$. This result is sharp.

Proof. Since $F$ is in $\delta^{*}, \operatorname{Re}\left[z F^{\prime}(z) / F(z)\right]>0$ for $|z|<1$. Thus there exists $\phi$, regular in $E$, such that $|\phi(z)| \leqq 1$ for $z$ in $E$ and such that

$$
\frac{z f(z)-\int_{0}^{z} f(t) d t}{\int_{0}^{z} f(t) d t}=\frac{z F^{\prime}(z)}{F(z)}=\frac{1-z \phi(z)}{1+z \phi(z)} .
$$

Thus

$$
f(z)=\frac{2}{z(1+z \phi(z))} \int_{0}^{z} f(t) d t .
$$

Therefore

$$
\begin{aligned}
\frac{z f^{\prime}(z)}{f(z)} & =\frac{-z \phi(z)-z^{2} \phi^{\prime}(z)}{1+z \phi(z)}+\frac{z f(z)-\int_{0}^{z} f(t) d t}{\int_{0}^{z} f(t) d t} \\
& =\frac{1-2 z \phi(z)-z^{2} \phi^{\prime}(z)}{1+z \phi(z)} .
\end{aligned}
$$

In order to determine where $f$ is starlike, we must determine those values of $z$ for which the real part of the right hand side of (1) is positive. This condition is equivalent to

$$
\operatorname{Re}\left[1-2 z \phi(z)-z^{2} \phi^{\prime}(z)\right][1+z \phi(z)]->0 .
$$

Condition (2) is equivalent to

$$
\operatorname{Re}\left[z^{2} \phi^{\prime}(z)\right][1+z \phi(z)]-<1-2|z|^{2}|\phi(z)|^{2}-\operatorname{Re}[z \phi(z)] .
$$

Using the well known result

$$
\left|\phi^{\prime}(z)\right| \leqq \frac{1}{1-|z|^{2}}\left(1-|\phi(z)|^{2}\right) \quad(|z|<1)
$$

and using the fact that $\operatorname{Re}[z \phi(z)] \leqq|z||\phi(z)|$, we see that condition (3) will be satisfied if

$$
\begin{aligned}
& \frac{|z|^{2}}{1-|z|^{2}}\left(1-|\phi(z)|^{2}\right)(1+|z||\phi(z)|) \\
& <(1-2|z||\phi(z)|)(1+|z||\phi(z)|) .
\end{aligned}
$$


Condition (4) is equivalent to

$$
2|z|^{2}+2|z||\phi(z)|\left(1-|z|^{2}\right)-|z|^{2}|\phi(z)|^{2}<1 .
$$

Thus, we need only show that condition (5) holds for all functions $\phi$, regular in $E$ and satisfying $|\phi(z)| \leqq 1$ for $z$ in $E$, provided $|z|<1 / 2$.

If in (5) we let $a=|z|$ and $x=|\phi(z)|$, then it is sufficient to show that for any fixed $a, 0 \leqq a<1 / 2$, the function $p(x)=2 a^{2}+2 a\left(1-a^{2}\right) x$ $-a^{2} x^{2}$ is bounded above by one for $0 \leqq x \leqq 1$. It is easily seen that $p^{\prime}(x)>0,0 \leqq x \leqq 1$, provided that $a<(\sqrt{ } 5-1) / 2$ and therefore if $a$ $<1 / 2$. Thus, if $0 \leqq a<1 / 2$, the maximum value of $p(x), 0 \leqq x \leqq 1$, is given by $q(a)=2 a+a^{2}-2 a^{3}$. Since $q^{\prime}(a)>0$ for $0 \leqq a<1 / 2, q(a)$ $<q(1 / 2)=1$ for $0 \leqq a<1 / 2$. Condition (2) is thus seen to be satisfied, if $|z|<1 / 2$. Hence $f$ is starlike for $|z|<1 / 2$.

To see that the result is sharp, let $F(z)=z /(1-z)^{2}$ which is in $\delta^{*}$. Then, $f(z)=z /(1-z)^{3}$ and $z f^{\prime}(z) / f(z)=(1+2 z) /(1-z)=0$ for $z=-1 / 2$. Thus, $f$ is not starlike in any circle $|z|<r$, if $r>1 / 2$.

Theorem 2. If $F$ is in $\Re$, then $f(z)=[1 / 2][z F(z)]^{\prime}$ is univalent in $E$ and is convex for $|z|<1 / 2$. This result is sharp.

Proof. We have $2 f^{\prime}(z)=2 F^{\prime}(z)+z F^{\prime \prime}(z)$. Thus

$$
2 \operatorname{Re}\left[\frac{f^{\prime}(z)}{F^{\prime}(z)}\right]=2+\operatorname{Re}\left[\frac{z F^{\prime \prime}(z)}{F^{\prime}(z)}\right] \text {. }
$$

Since $F$ is in $\Re$, the right hand side of (6) is positive in $E$. Thus, $f$ is close-to-convex relative to $F$ and therefore is univalent in $E$.

To show that $f$ is convex for $|z|<1 / 2$, we notice that $z f^{\prime}(z)=[1 / 2]$ $\cdot\left[z\left(z F^{\prime}(z)\right)\right]^{\prime}$. Since $F$ is in $\Re, z F^{\prime}$ is in $\delta^{*}$. Therefore, by Theorem 1 , $z f^{\prime}$ is starlike for $|z|<1 / 2$ and thus $f$ is convex for $|z|<1 / 2$.

To see that the result is sharp, let $F(z)=z /(1-z)$ which is in $\mathcal{K}$. Then $f(z)=\left(2 z-z^{2}\right) / 2(1-z)^{2}$ and $1+\left[z F^{\prime \prime}(z) / F^{\prime}(z)\right]=(1+2 z) /(1-z)$ $=0$ for $z=-1 / 2$. Therefore $f$ is not convex in any circle $|z|<r$, if $r>1 / 2$.

TheOREM 3. If $F$ is in e, then $f(z)=1 / 2[z F(z)]^{\prime}$ is close-to-convex for $|z|<1 / 2$. This result is sharp.

Proof. Since $F$ is in $\mathcal{e}$, there exists $G$ in $\mathcal{S}^{*}$ such that

$$
\operatorname{Re}\left[\frac{z F^{\prime}(z)}{G(z)}\right]>0 \quad(|z|<1) .
$$

Let $g(z)=[1 / 2][z G(z)]^{\prime}$, then, by Theorem $1, g$ is starlike for $|z|<1 / 2$. To prove the theorem, it is sufficient to show that $\operatorname{Re}\left[z f^{\prime}(z) / g(z)\right]>0$ for $|z|<1 / 2$. We have 


$$
\frac{z F^{\prime}(z)}{G(z)}=\frac{z f(z)-\int_{0}^{z} f(t) d t}{\int_{0}^{z} g(t) d t} .
$$

Thus, by (7), we may set

$$
\frac{z f(z)-\int_{0}^{z} f(t) d t}{\int_{0}^{z} g(t) d t}=P(z)
$$

where $P$ is regular in $E$ and satisfies $P(0)=1$ and $\operatorname{Re}(P(z))>0$ for $z$ in $E$. We thus have

$$
z f^{\prime}(z)=P(z) g(z)+P^{\prime}(z) \int_{0}^{z} g(t) d t .
$$

Therefore

$$
\frac{z f^{\prime}(z)}{g(z)}=P(z)+P^{\prime}(z)\left[\frac{\int_{0}^{z} g(t) d t}{g(z)}\right] .
$$

Using the known result [1], [3], [6]

$$
\left|P^{\prime}(z)\right| \leqq \frac{2 \operatorname{Re}[P(z)]}{1-|z|^{2}} \quad(|z|<1)
$$

we have from (10)

$$
\operatorname{Re}\left[\frac{z f^{\prime}(z)}{g(z)}\right] \geqq \operatorname{Re}[P(z)]\left[1-\frac{2}{1-|z|^{2}}\left|\frac{\int_{0}^{z} g(t) d t}{g(z)}\right|\right] .
$$

Moreover

$$
\frac{z g(z)}{\int_{0}^{z} g(t) d t}=\frac{[1 / 2]\left(z[z G(z)]^{\prime}\right)}{[1 / 2](z G(z))}=1+\frac{z G^{\prime}(z)}{G(z)} .
$$

Since $G$ is in $\mathcal{S}^{*}, \operatorname{Re}\left[z G^{\prime}(z) / G(z)\right]>0$ for $z$ in $E$. Thus $\operatorname{Re}[z g(z)$ $\left./ \int_{0}^{z} g(t) d t\right]>1$ for $z$ in $E$. Hence, there exists $\phi$, regular in $E$ and satisfying $|\phi(z)| \leqq 1$ for $z$ in $E$, such that $z g(z) / \int_{0}^{z} g(t) d t=$ 
$2 /(1+z \phi(z))$. Therefore

$$
\left|\frac{\int_{0}^{z} g(t) d t}{g(z)}\right|=\left|\frac{z+z^{2} \phi(z)}{2}\right| \leqq \frac{1}{2}\left(|z|+|z|^{2}\right) .
$$

Combining (11) and (12) we have

$$
\begin{aligned}
\operatorname{Re}\left[\frac{z f^{\prime}(z)}{g(z)}\right] & >\operatorname{Re}[P(z)]\left[1-\frac{|z|+|z|^{2}}{1-|z|^{2}}\right] \\
& =\operatorname{Re}[P(z)]\left[\frac{1-2|z|}{1-|z|}\right] .
\end{aligned}
$$

The right hand side of (13) is positive provided $|z|<1 / 2$.

To see that the result is sharp, let $F(z)=z /(1-z)^{2}$ which is in $\delta^{*}$ and therefore in $\mathcal{C}$. Then $f(z)=z /(1-z)^{3}$ and $f^{\prime}(z)=(1+2 z) /(1-z)^{4}$ $=0$ for $z=-1 / 2$. Thus, $f(z)$ is not univalent and therefore not closeto-convex in $|z|<r$, if $r>1 / 2$.

An interesting subclass of $\mathfrak{e}$ is that class made up of functions $F$ which satisfy $\operatorname{Re}\left[F^{\prime}(z)\right]>0$ for $z$ in $E[3]$. Theorem 3 can be improved for this subclass.

TheOREM 4. Let $F$ be such that $\operatorname{Re}\left[F^{\prime}(z)\right]>0$ for $z$ in $E$ and let $f(z)=[1 / 2][z F(z)]^{\prime}$, then $\operatorname{Re}\left[f^{\prime}(z)\right]>0$ for $|z|<(\sqrt{ } 5-1) / 2$. This result is sharp.

Proof. Let $F^{\prime}(z)=P(z)$ where $P(0)=1$ and $\operatorname{Re}(P(z))>0$ for $z$ in $E$. We then have

$$
2 f^{\prime}(z)=2 F^{\prime}(z)+z F^{\prime \prime}(z)=2 P(z)+z P^{\prime}(z) .
$$

Using again the fact that $\left|P^{\prime}(z)\right| \leqq 2 \operatorname{Re}[P(z)] /\left[1-|z|^{2}\right]$ for $z$ in $E$, we have

$$
\begin{aligned}
2 \operatorname{Re}\left[f^{\prime}(z)\right] & \geqq 2 \operatorname{Re}[P(z)]-|z|\left|P^{\prime}(z)\right| \\
& \geqq 2 \operatorname{Re}[P(z)]\left[1-\frac{|z|}{1-|z|^{2}}\right] \\
& =2 \operatorname{Re}[P(z)]\left[\frac{1-|z|-|z|^{2}}{1-|z|^{2}}\right] .
\end{aligned}
$$

The right hand side of (14) is positive provided $|z|<(\sqrt{ } 5-1) / 2$.

To see that the result is sharp, let $F(z)=-z-2 \log (1-z)$. Then $f(z)=[1 / 2]\left[2 z^{2} /(1-z)-2 \log (1-z)\right]$ and $f^{\prime}(z)=\left(1+z-z^{2}\right) /(1-z)^{2}$ $=0$ for $z=(1-\sqrt{ } 5) / 2$. 


\section{REFERENCES} 158.

1. R. J. Libera, Some radius of convexity problems, Duke Math. J. 31 (1964), 143-

2. - Some classes of regular univalent functions, Proc. Amer. Math. Soc. 16 (1965), 755-758.

3. T. H. MacGregor, Functions whose derivative has a positive real part, Trans. Amer. Math. Soc. 104 (1962), 532-537.

4. - The radius of convexity of starlike functions of order 1/2, Proc. Amer. Math. Soc. 14 (1963), 71-76.

5. R. M. Robinson, Univalent majorants, Trans. Amer. Math. Soc. 61 (1947), 1-35.

6. M. S. Robertson, Extremal problems for analytic functions with positive real part and applications, Trans. Amer. Math. Soc. 106 (1963), 236-253.

Lafayette College 